\title{
Expertise psychiatrique et justice pénale
}

\section{Jean Martin}

Dr méd., membre de la rédaction

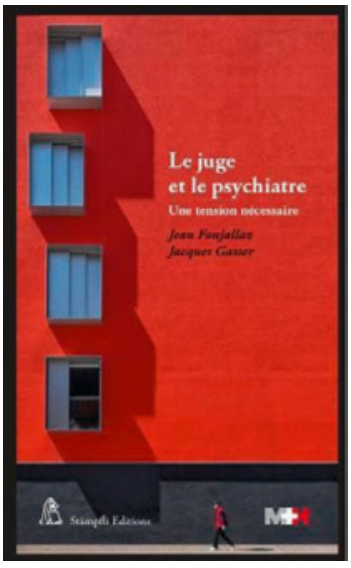

Jean Fonjallaz; Jacques Gasser Le juge et le psychiatre

Une tension nécessaire Editions: Médecine et Hygiène, Chêne-Bourg - Suisse; Stämpfli Editions, Berne; 2017 ISBN: 978-2880494254 223 Pages. 29 CHF.

Jean Fonjallaz est docteur en droit et juge fédéral. Jacques Gasser est professeur à la Faculté de médecine de Lausanne et directeur du Département de psychiatrie du CHUV; au cours de sa carrière, il s'est beaucoup occupé d'expertises. Ils publient un livre susceptible de répondre à des manques persistants - malgré des améliorations dans le passé récent - dans la formation des professionnels impliqués, du domaine juridique (procureurs, juges, avocats) et du domaine médical. «Il était une fois, à la fin du $\mathrm{XX}^{\mathrm{e}}$ siècle, des disputes entre juges et psychiatres. Par nécessité, les auteurs du présent ouvrage ont engagé un dialogue qui leur a permis de faire le constat de leurs propres méconnaissances croisées et de tenter de répondre à un flot de questions.» Ceci sur les conditions et impératifs de leurs rôles respectifs en matière pénale, notamment lorsque se posent des questions sur la responsabilité des prévenus. «Les représentants du ministère public et les parties jouent sur un terrain qui n'est pas celui de l'expert, qui lui procède à un travail fondé sur la connaissance et la réflexion médico-scientifiques.» Répondant aussi, dans la foulée, aux souhaits d'information du public intéressé par le fonctionnement de la justice, thème qui prend de la place dans les média. "Le juge et le psychiatre» est un ouvrage clair, bien structuré, "d'introduction" disent les auteurs, mais qui néanmoins couvre largement le champ, apportant une foule d'informations utiles: dispositions légales, règles pertinentes, modes de faire de différentes instances et ni- veaux, avec des éléments aussi sur les «mises en scène» $\mathrm{y}$ relatives.

\section{Quatre parties}

La première est "Le procès pénal» (moitié du livre), décrivant les sources du droit, les acteurs du procès et son déroulement, les sanctions et mesures; mesures qui sont envisagées "si une peine seule ne peut écarter le danger d'autres infractions». Elles visent deux types de personnes, qui d'ailleurs ne devraient pas nécessairement être traités de manière identique: celles qui ont besoin d'un traitement médical, d'une part, et d'autre part celles qu'il se justifie de priver de leur liberté car elles représentent un danger trop grand pour la société (notion d'internement).

Les trois suivantes ont pour titres «L'expert psychiatre dans le procès pénal", "Comment se construit une expertise psychiatrique?» et «Le juge et le psychiatre, alliés ou adversaires?». En complément, une bibliographie choisie, le texte des normes légales concernées et un exemple-type de questionnaire auquel la justice pénale souhaite qu'un expert réponde.

Rappel: «Les plus anciennes législations considéraient la folie comme un motif d'exemption de punition (...) On estimait que l'on ne pouvait pas imputer une faute à quelqu'un dont l'esprit est aliéné et il en allait de même pour l'enfant, l'animal ou la tuile qui tombe d'un toit." Les choses ont changé et les questions de responsabilité (entière, restreinte ou nulle) se sont complexifiées, avec l'élaboration et la diversification des codes pénaux. Evolution influencée aussi par l'accent croissant mis sur le respect des droits humains. Les citations ci-dessous illustrent des points majeurs.

\section{Changement de paradigme}

«En une quinzaine d'années, la justice pénale - du moins le législateur - est passé d'une logique de punition et de réinsertion sociale à un modèle de gestion du risque mettant au centre la protection de la société.» L'intérêt des milieux professionnels, politiques et médiatiques s'est déplacé de la problématique de la res- 
ponsabilité à celle du risque de récidive, avec une intensification des réflexions sur la dangerosité.

«Ceci est à la racine des difficultés des experts psychiatres qui doivent se prononcer non seulement sur ce qu'ils savent faire (diagnostiquer, proposer des thérapeutiques, apprécier la responsabilité), mais également sur des aspects beaucoup plus flous et éloignés de leurs préoccupations habituelles, qui sont ceux relevant de la sécurité publique.» Il n'en reste pas moins que la maîtrise de la sécurité constitue une préoccupation forte, en particulier celle de savoir quels sont les risques acceptables - si l'on admet que le risque zéro n'est pas réalisable.

\section{A propos de l'expertise et de son rapport}

«En premier lieu, l'expertisé doit être averti que le médecin n'est pas dans son rôle habituel de thérapeute mais qu'il agit sur mandat d'une autorité judiciaire à laquelle il devra communiquer tous les éléments nécessaires, et qu'il n'est donc pas soumis au secret médical dans cette fonction.»

\section{En quinze ans, on est passé d'une logique de punition et de réinsertion à un modèle de gestion du risque et de la dangerosité}

«La règle essentielle est que le travail d'expert ne devrait jamais être un travail solitaire, ce qui se distingue des anciennes pratiques. Dans l'expertise à deux, il y a lieu de mettre en place un processus de collaboration (...). Adopter une méthode qui valorise le consensus dans la prise de décision, en favorisant la négociation et la création d'un point de vue nouveau. Ceci permet de travailler les éventuels désaccords, non pas pour les gommer, mais pour les expliciter et trouver la meilleure solution.»

«Dans le cas où la façon dont les fait sont survenus jouerait un rôle quant aux réponses à donner, l'expert doit prendre en compte séparément les diverses possibilités. Ce n'est pas à lui de choisir la 'meilleure' version. Il peut ainsi être amené à donner deux versions de ses conclusions en fonction des 'réalités' différentes décrites par l'accusation et le prévenu.»

«La discussion (chapitre du rapport) est la partie la plus importante de l'expertise psychiatrique. Elle représente l'interface entre le monde médical et le monde juridique. C'est là qu'il s'agit de chercher s'il existe un lien de causalité entre les éléments cliniques observés et les actes reprochés à l'expertisé, ce qui revient à mettre en évidence un éventuel déterminisme pathologique. L'expert doit transposer ses observations en langage compréhensible pour les non-spécialistes.»
«Il est important de savoir que l'expert psychiatre est formé à se connaitre, en particulier à connaître ses réactions vis-à-vis des autres, pour faire la part des choses entre les siennes propres et celles qui viennent de l'autre. Un des instruments de travail du psychiatre est sa connaissance de lui-même.»

\section{Des considérations de fond}

«Le droit pénal est fondé sur la conception que chacun est en principe libre d'agir. C'est le libre arbitre, la conscience et la volonté sont présumées (...) En revanche la psychiatrie a observé que le comportement d'un individu est conditionné par différents éléments liés à son état mental et à son histoire. C'est le déterminisme». Aussi: «Le droit fonctionne le plus souvent par catégories et concepts distincts, alors que les psychiatres voient leurs observations dans une certaine globalité et un certain continuum" - c'est J.M. qui souligne; en tant que médecin cantonal, j'ai bien souvent constaté, et eu à expliquer, comment le droit voit/tend à voir les choses en noir ou blanc, alors que le médecin (avec les autres soignants, les travailleurs sociaux ...) voit - est contraint de voir! - que la vie n'est faite que de nuances de gris, du plus clair au plus foncé.

Est-ce que comprendre, c'est excuser ? Excellente question posée à la fin du livre. «Trop souvent, le travail de compréhension du comportement d'une personne est interprété comme excusant ce comportement, avec pour effet, ou pire pour but, de réduire la punition.» On confond là deux choses. "Comprendre est de l'ordre de la connaissance, c'est l'action qui vise à accéder au sens, à chercher les mécanicismes ou déterminants significatifs d'un acte (...) En aucune façon le psychiatre ne devra se prononcer sur la valeur positive ou négative de l'acte. Sanctionner, en revanche, est une action d'un autre ordre, qui a pour fin d'appliquer la loi que s'est donnée la collectivité.»

«Sans psychiatrie, on reviendrait à un cadre légal où ce ne serait plus l'être humain mais bien l'acte pénal qui serait au centre de la réflexion amenant à fixer la sanction. Il serait alors difficile d'échapper à la règle du 'tarif' pénal, qui ne serait sans doute pas conforme aux aspirations d'une société fondée sur le respect des droits humains.»

En guise de conclusion: «Cet ouvrage se veut l'illustration que le dialogue entre un juge et un psychiatre est susceptible de déboucher sur un résultat concret et utile, aux confins de deux matières dont les fondements et fonctionnements sont diamétralement divergents mais dont l'interaction devrait servir le vivre ensemble.» 